\title{
Superacid-Promoted Reactions of Pyrazole- carboxaldehydes and the Role of Dicationic Electrophiles
}

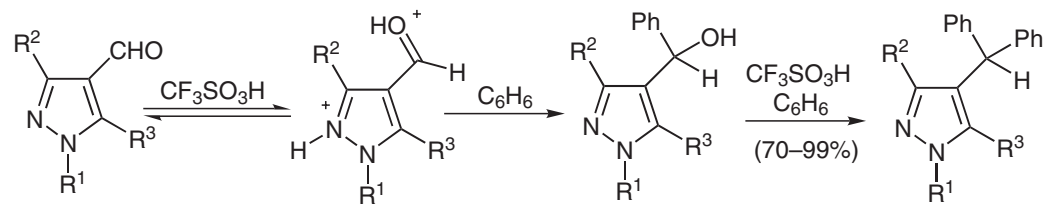

Significance: Treatment of pyrazolecarboxaldehydes with super acids leads to the formation of dicationic species which are highly reactive for electrophilic diarylation even with weakly nucleophilic arenes. Thus a variety of substituted pyrazolecarboxaldehydes condense with benzene to provide side-chain substituted products. The mechanism involving a dicationic intermediate is supported by low temperature ${ }^{13} \mathrm{C}$ NMR studies.
Comment: Friedel-Crafts type electrophilic reactions of aromatics with benzaldehyde derivatives are a classical method for hydroxyarylation and has been used in large-scale industrial synthesis, e.g. DDT. (J. March Advanced Organic Chemistry, 5th ed.; Wiley: New York, 2001, p 719). This work builds on the arguably poorly appreciated fact that protonated $\mathrm{N}$-heterocycles can enhance the reactivity of adjacent electrophilic centers such as aldehydes for hydroxyarylation and further arylation. Strong acid-catalyzed intramolecular FriedelCrafts reactions are also reported for some substituted pyrazoles. 